\title{
Randomized clinical trial to assess the effectiveness of remote patient monitoring and physician care in reducing office blood pressure
}

\author{
Yoon-Nyun Kim ${ }^{1,5}$, Dong Gu Shin ${ }^{2,5}$, Sungha Park ${ }^{3}$ and Chang Hee Lee ${ }^{4}$
}

The effectiveness of remote patient monitoring and physician care for the treatment of hypertension has not been demonstrated in a randomized clinical trial. The objective of this study was to evaluate the effectiveness of remote patient monitoring with or without remote physician care in reducing office blood pressure in patients with hypertension. A total of $\mathbf{3 7 4}$ hypertensive patients over 20 years of age were randomized into the following three groups: group (1) control, the patients received usual clinical care with home BP monitoring; group (2) the patients were remotely monitored and received office follow-up; and group (3) the patients received remote monitoring without physician office care using the remote monitoring device. For each group, inoffice follow-up care was scheduled every 8 weeks for 24 weeks. The primary end point was the difference in sitting SBP at the 24-week follow-up. No difference between the three groups was observed in the primary end point (adjusted mean sitting SBP was as follows: group 1: $-8.9 \pm 15.5 \mathrm{~mm} \mathrm{Hg}$, group 2: $-11.3 \pm 15.9 \mathrm{~mm} \mathrm{Hg}$, group 3: $-11.6 \pm 19.8 \mathrm{~mm} \mathrm{Hg}$, (NS). Significant differences in achieving the target BP at the 24th week of follow-up were observed between groups 1 and 2 . The subjects over 55-years old had a significant decrease in the adjusted mean sitting SBP in groups 2 and 3 compared with that of the control group. Remote monitoring alone or remote monitoring coupled with remote physician care was as efficacious as the usual office care for reducing blood pressure with comparable safety and efficacy in hypertensive patients.

Hypertension Research (2015) 38, 491-497; doi:10.1038/hr.2015.32; published online 19 March 2015

Keywords: blood pressure; home blood pressure; remote monitoring; remote physician care

\section{INTRODUCTION}

Although achieving the target blood pressure (BP) is the most important prognostic factor for the treatment of hypertension, the latest NHANES 2007-2008 survey showed that only 50.1\% of all hypertensive patients met this objective. ${ }^{1}$ Numerous factors have been shown to be associated with improved BP control. Critical among these factors is the degree of patient awareness and adherence to the treatment. A previous study demonstrated that self measurement of home BP is associated with improved treatment compliance, indicating that self measurement might help increase treatment adherence. ${ }^{2}$ Recent studies have shown that telemonitoring of home BP might be more effective than conventional treatment in achieving target BP. 3,4 To date, no randomized clinical trial has compared the efficacy of conventional home BP monitoring to a web-based remote patient monitoring device. The clinical trial presented here had two major objectives. First, to determine whether remote monitoring homebased BP monitoring is more efficacious in reducing office BP compared with conventional treatment with home BP measurement.
Second, to determine whether there is a difference in blood pressure lowering according to web-based remote patient monitoring with or without remote physician care (LG Smart Care system, LG Electronics, Seoul, Korea).

\section{METHODS}

Study design

This study was a multi-center, randomized, parallel, interventional, open-label trial involving the following clinical centers in South Korea: Yonsei University, Yeungnam University and Keimyung University (Clinical Trials GOV NCT 01335984). The clinical trial was performed between 20 December 2010 and 3 April 2013. The remote monitoring system used in this study was the LG Smart Care system.

The eligible participants were assigned to the three groups with equal probability according to the randomization code. Because this study is an openlabeled trial, blinding was not performed. The group 1 (control) patients $(n=124)$ received typical clinical care using home BP monitoring validated with the BHS protocol (UA 767PBT, A\&D medical, Tokyo, Japan) with no remote monitoring and office follow-up (FU) visits at 8-week intervals for

\footnotetext{
${ }^{1}$ Department of Internal Medicine, Division of Cardiology, Keimyung University College of Medicine, Daegu, Korea; ${ }^{2}$ Department of Internal Medicine, Cardiovascular Division, Yeungnam University college of medicine, Daegu, Korea; ${ }^{3}$ Divison of Cardiology, Severance Cardiovascular Hospital and Cardiovascular Research Institute, Yonsei University College of Medicine, Seoul, Korea and ${ }^{4}$ LG Electronics, Seoul, Korea

${ }^{5}$ These authors contributed equally to this work.

Correspondence: Dr S Park, Division of Cardiology, Severance Cardiovascular Hospital, Yonsei University College of Medicine, 50-1 Yonsei-ro, Seodaemun-gu, Seoul 120-752, Korea. E-mail: shpark0530@yuhs.ac

Received 30 August 2014; revised 22 January 2015; accepted 26 January 2015; published online 19 March 2015
} 
24 weeks. ${ }^{5}$ The subjects were instructed to measure and record their home $\mathrm{BP}$ measurement in their diary and bring the data to each office visit. The subjects were instructed on the proper intake of their medications, as well as maintenance of their usual healthy lifestyle modifications of their dietary and exercise habits. The group $2(n=124)$ patients received remote monitoring of home BP through the LG Smart Care system; however, they did not receive remote physician care. These patients receive in-office physician care at FU appointments every 8 weeks for 24 weeks. The group $3(n=126)$ patients received remote monitoring of home $\mathrm{BP}$ and remote physician care without inoffice physician care. Remote physician care was provided every 8 weeks for 24 weeks by the attending physician of the subject. For the remote monitoring, routine contact was made with the patient every week from the 1st to 9th week and once every 4 weeks from the 10th to the 24th week. In addition, the patient was contacted in the following cases: (i) when the 1-week average home BP was $\geqslant 160 / 100 \mathrm{~mm} \mathrm{Hg}$; (ii) when the measured BP was $\geqslant 180 / 120 \mathrm{~mm} \mathrm{Hg}$; and (iii) if the patient did not measure the BP at home for 3 consecutive days. The patients were instructed to measure the BP at home in the dominant arm, once in the morning and once in the evening. The patients enrolled in the remote monitoring arm (group 2, group 3) were educated regarding the use of the supplied netbook computer and the LG Smart Care system. All the data were uploaded onto the server by Bluetooth and constantly monitored by healthcare professionals (physicians, nurses, a nutritionist, an exercise trainer) involved in the study. In addition to the BP data that was obtained, the patients were instructed to upload the records of their daily dietary intake, as well as the types and duration of the exercise programs, which were monitored by the nutritionist and the exercise trainer. The adherence to anti-hypertensive medications was assessed by records in a medication diary provided to the patients at the time of enrollment. The adherence was calculated by the number of days anti-hypertensive medications were taken, divided by the study duration.

Ambulatory BP was measured in the non-dominant arm using a validated ambulatory BP monitoring (ABPM) device (A\&D TM-2430, A\&D Medical, San Jose, CA, USA). The daytime average BP was defined as the average of the BP measurements obtained from 0700 hours to 2259 hours. The night time average BP was defined as the average of the BP measurements obtained from 2300 hours to 0659 hours. The white-coat effect was defined as a baseline daytime average systolic/diastolic ABPM $<135 / 85 \mathrm{~mm} \mathrm{Hg}$ (130/80 for DM and/or CKD), respectively. For the patients enrolled in the web-based monitoring-only arm (group 2), an in-person office visit to the attending physician was required every 8 weeks. The attending physicians cared for the patient based upon the data obtained through the remote patient monitoring, as well as the data obtained in the office. For the patients enrolled in the remote monitoring/remote treatment arm (group 3), the attending physician assessed the status of the patient by remote monitoring. Anti-hypertensive medications were adjusted and prescribed by the attending physicians according to the patient's data. The hypertension treatment regimen, based on the major clinical guidelines, was left to the discretion of the attending physician. ${ }^{6-8}$ For the remote physician monitoring arm, the necessary medications were delivered to the patient.

The study protocol and informed consent form were reviewed and approved by the Institutional Review Board of Yonsei University Shinchon Severance Hospital, Yeungnam University Hospital and Keimyung University Hospital. All the procedures were performed in accordance with the institutional guidelines. All the patients provided informed consent prior to being enrolled.

\section{Inclusion and exclusion criteria \\ Inclusion criteria}

1. Hypertensive patients over 20 years of age whose office BP was uncontrolled with one or more anti-hypertensive medications (systolic BP (SBP) $\geqslant 140$ $\mathrm{mm} \mathrm{Hg}$; for diabetes mellitus (DM) or chronic kidney disease (CKD), $\mathrm{SBP} \geqslant 130 \mathrm{~mm} \mathrm{Hg}$ ).

\section{Exclusion criteria}

1. The patients with an average sitting SBP of $>200 \mathrm{~mm} \mathrm{Hg}$ at the screening visit.

2. Secondary hypertension.

3. HbAlc > $11 \%$.

4. Hypertensive patients requiring admission care.

5. CKD with a serum creatinine level of $>1.5$ times the upper limit of normal.

6. Chronic liver disease with aspartate aminotransferase/alanine aminotransferase over three times the upper limit of normal.

7. History of acute myocardial infarction, acute coronary syndrome, congestive heart failure and/or valvular heart disease within the last 6 months.

8. Chronic debilitating illness.

9. Autoimmune disease.

10. Patients taking medications that might influence BP (sedatives, hypnotics, lithium, selective serotonin reuptake inhibitors, tricyclic antidepressants, nonsteroidal anti-inflammatory drugs, oral contraceptives, alpha agonists, steroids).

11. Patients with known allergies to angiotensin-converting-enzyme inhibitors or angiotensin receptor blockers.

12. Patients with a history of alcoholism.

\section{Statistical analysis}

The study was initially designed to enroll 564 patients (under the assumption of a $25 \%$ dropout rate), which was later revised to enroll 495 patients equally to each of the three intervention groups, under the assumption of a $20 \%$ dropout rate because the dropout rate was lower than expected (Figure 1). With a sample size of 165 patients per group, the trial had $80 \%$ power to detect ( $\alpha=0.05$; two-sided test) a between-treatment difference between the group 1 and groups 2 and 3 of $3.8 \mathrm{~mm} \mathrm{Hg}$ in the SBP change from baseline with a s.d. for the SBP of $11.43 \mathrm{~mm} \mathrm{Hg}$, with a $20 \%$ dropout rate. The between-treatment difference of $3.8 \mathrm{~mm} \mathrm{Hg}$ and an s.d. of $11.43 \mathrm{~mm} \mathrm{Hg}$ were estimated from similar hypertension trials. ${ }^{9-11}$

The primary end point was the difference in the sitting SBP at the 24th week of FU by the intention-to-treat (ITT) analysis compared with the baseline measurement. The secondary end points were as follows: (i) the difference in the sitting diastolic BP (DBP) at the 24th week of FU by the ITT analysis compared with the initial measurement; (ii) the percentage of patients achieving the target BP $(<140 / 90 \mathrm{~mm} \mathrm{Hg}$ and $<130 / 80$ for patients with $\mathrm{DM}$ and/or CKD); (iii) the difference in the $24 \mathrm{~h}$ ambulatory BP at the 24 th week of FU by the ITT analysis compared with the initial measurement; (iv) the percentage of patients achieving the target daytime ambulatory BP $(<135 / 85$ $\mathrm{mm} \mathrm{Hg},<130 / 80$ for $\mathrm{DM}$ and/or CKD); (v) the difference in the average home SBP/DBP at the 24th week of FU by the ITT analysis compared with the initial measurement; and (vi) drug adherence ((the number of days taking antihypertensive medications/the number of days enrolled in the clinical trial) $\times$ 100 ). A subgroup analysis according to age (55 years), gender and the presence of DM/CKD was performed.

For the statistical analysis, the baseline characteristics were analyzed using analysis of variance for the continuous variables and the $\chi^{2}$ method for the discrete variables. The difference in the adjusted mean BP reduction was assessed using the analysis of covariance. The statistical analysis was performed using SAS 9.1 software (SAS Institute, Cary, NC, USA).

\section{RESULTS}

A total of 440 patients were screened for the study. Among the 440 subjects (66 failed to meet the inclusion criteria), 374 patients were randomized into three groups and analyzed by an ITT analysis (group 1: 124, group 2: 124, group 3: 126). Among the 374 patients initially randomized, 331 patients were available for the per-protocol analysis (group 1: 115, group 2: 111, group 3: 105, Figure 1). There were no significant differences between the three groups in terms of age, gender, the body mass index, smoking or alcohol intake (Table 1). 


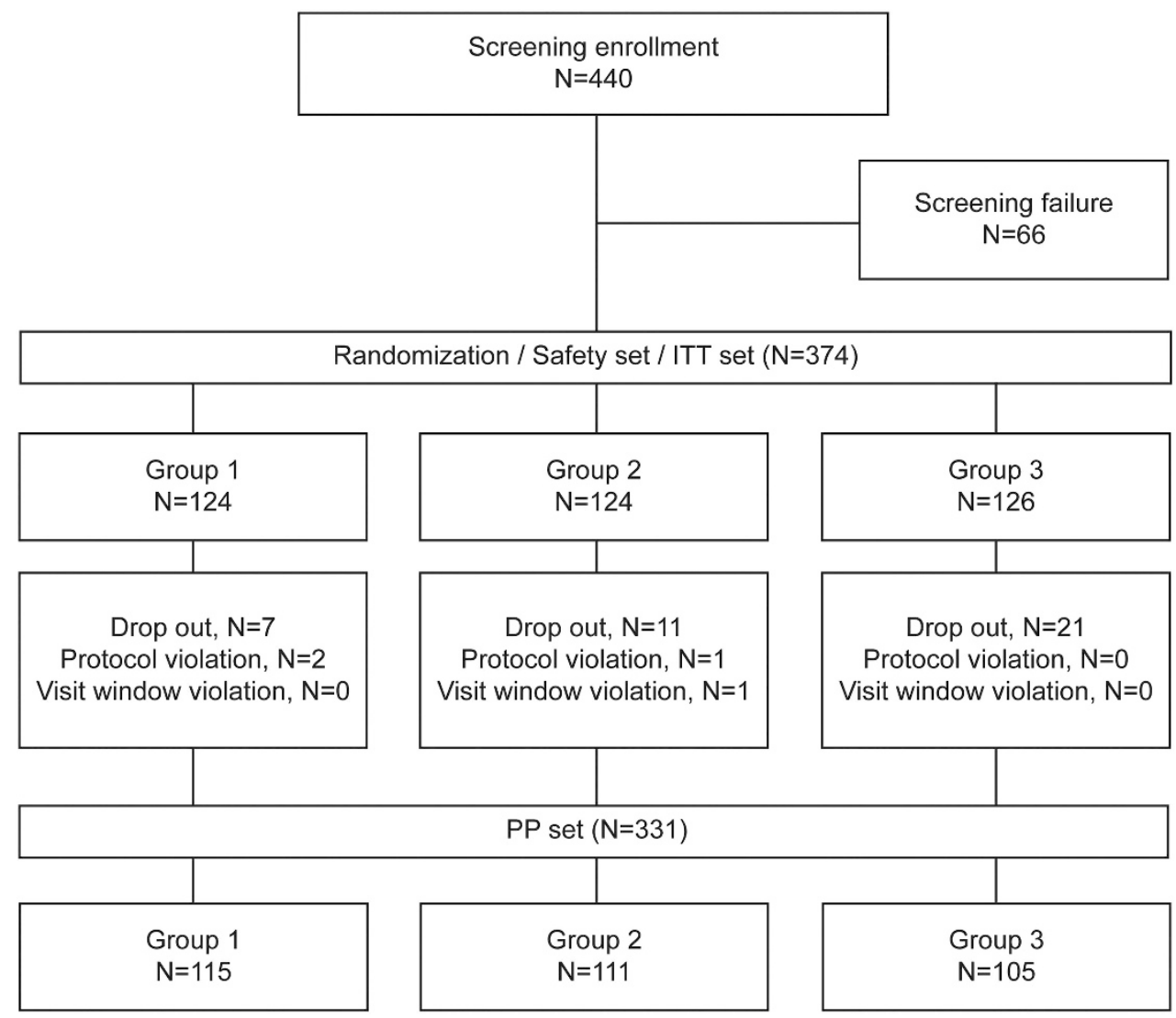

Figure 1 Disposition of study subjects.

Table 1 Baseline characteristics of patients (ITT set)

\begin{tabular}{|c|c|c|c|c|}
\hline \multirow[b]{3}{*}{ Characteristics } & \multicolumn{3}{|c|}{ ITT set $(\mathrm{n}=374)$} & \multirow[b]{3}{*}{ P-value } \\
\hline & \multirow[b]{2}{*}{$\begin{array}{l}\text { Group } 1 \\
(\mathrm{n}=124)\end{array}$} & \multicolumn{2}{|c|}{ Smartcare service } & \\
\hline & & $\begin{array}{l}\text { Group } 2 \\
(\mathrm{n}=124)\end{array}$ & $\begin{array}{l}\text { Group } 3 \\
(n=126)\end{array}$ & \\
\hline Age (years) & $58.8 \pm 10.6$ & $56.4 \pm 9.9$ & $56.1 \pm 11$ & 0.09 \\
\hline$>55 y(n, \%)$ & $77(62.1)$ & $75(60.5)$ & 78 (61.9) & 0.96 \\
\hline Males $(n, \%)$ & 71 (57.3) & $67(54.0)$ & $79(62.7)$ & 0.37 \\
\hline $\begin{array}{l}\text { Body mass index } \\
\left(\mathrm{kg} \mathrm{m}^{-2}\right)\end{array}$ & $25.8 \pm 3.1$ & $26.1 \pm 3.8$ & $25.9 \pm 3.4$ & 0.80 \\
\hline \multicolumn{5}{|l|}{ Smoke (n, \%) } \\
\hline Smoker & $19(15.3)$ & $16(12.9)$ & $27(21.4)$ & 0.39 \\
\hline Former smoker & $37(29.8)$ & $37(29.8)$ & $39(31.0)$ & \\
\hline Non smoker & $68(54.8)$ & $71(57.3)$ & $60(47.6)$ & \\
\hline Drinker $(n, \%)$ & $62(50)$ & $65(52.4)$ & $78(61.9)$ & 0.14 \\
\hline $\begin{array}{l}\text { Hypertension } \\
\text { duration (m) }\end{array}$ & $8.4 \pm 6.8$ & $8.9 \pm 8$ & $7.2 \pm 6.4$ & 0.27 \\
\hline $\mathrm{DM}(n, \%)$ & $11(8.8)$ & $21(16.9)$ & $16(12.7)$ & 0.16 \\
\hline $\operatorname{CKD}(n, \%)$ & $1(0.8)$ & $2(1.6)$ & $1(0.8)$ & 0.85 \\
\hline
\end{tabular}

Abbreviations: CKD, chronic kidney disease; DM, diabetes mellitus; ITT, intention-to-treat. Data are shown as mean \pm s.d.

a $P$-value from an ANOVA for continuous outcomes and $\chi 2$ (or Fisher's exact) test for binary outcomes comparing a difference between any of the three study groups.
No significant differences were observed among the three groups in terms of the baseline SBP (Table 2, $P=$ non significant). There were no significant differences in the $24 \mathrm{~h}$ ambulatory BP, daytime ambulatory BP or night time ambulatory BP (Supplementary Table 1). Among the subjects who underwent measurement of the baseline ABPM data, no significant differences in the proportion of subjects with the white-coat effect were observed (Supplementary Table 1). The analysis of the primary end point revealed that there were no significant differences between the three groups in terms of the decrease in the adjusted mean sitting SBP at the 24th week in the FU (group 1: $-8.9 \pm 15.5 \mathrm{~mm} \mathrm{Hg}$, group 2: $-11.3 \pm 15.9 \mathrm{~mm} \mathrm{Hg}$, group 3: $-11.6 \pm 19.8 \mathrm{~mm} \mathrm{Hg}, P$-value $=$ non significant, Table 2). There was a significant difference between the groups 1 and 2 in terms of achieving the target BP (SBP and DBP) at the 24th week of FU (Table 3). The subgroup analysis revealed that in the subjects over the age of 55, there was a significant decrease in the adjusted mean sitting SBP in the groups 2 and 3 compared with that in the control group (Table 2). Among the subjects over the age of 55, the patients in the group 2 and 3 achieved the target BP by the 24th week of FU at a significantly higher rate than the patients in group 1 (Table 3). Baseline and FU ABPM was performed in 96/124 subjects in group 1 (77.4\%), 89/124 (71.8\%) in group 2 and 87/126 (69.1\%) in group 3. The analysis of the ABPM data in the subjects with true uncontrolled hypertension showed a significant difference in terms of the changes in the ABPM SBP compared with the baseline within all three groups at FU (Table 4). There were no significant differences in the change in the ABPM SBP among the groups (Table 4). When the average 1-week home BP measurements (excluding the 1st day of measurement) were compared, the patients in the group 3 demonstrated a significant 


\begin{tabular}{|c|c|c|c|c|c|c|}
\hline & \multicolumn{3}{|c|}{ ITT set $(\mathrm{n}=374)$} & \multicolumn{3}{|c|}{ P-values for difference between groups ${ }^{b}$} \\
\hline & \multirow[b]{2}{*}{ Group $1(\mathrm{n}=124)$} & \multicolumn{2}{|c|}{ Smartcare services } & \multirow[b]{2}{*}{ Group 1 vs. 2} & \multirow[b]{2}{*}{ Group 1 vs. 3} & \multirow[b]{2}{*}{ Group 2 vs. 3} \\
\hline & & Group $2(\mathrm{n}=124)$ & Group $3(\mathrm{n}=126)$ & & & \\
\hline \multicolumn{7}{|l|}{ Systolic BP } \\
\hline Baseline & $143.2 \pm 13$ & $142.9 \pm 14.5$ & $143.1 \pm 14.7$ & & & \\
\hline Week 24 & $134.2 \pm 14.5$ & $130.7 \pm 15$ & $132 \pm 15.6$ & & & \\
\hline Change $^{\mathrm{C}}$ & $-8.9 \pm 15.5$ & $-11.3 \pm 15.9$ & $-11.6 \pm 19.8$ & 0.11 & 0.52 & 0.65 \\
\hline$P$-values for differences within group ${ }^{d}$ & $<0.01$ & $<0.01$ & $<0.01$ & & & \\
\hline
\end{tabular}

Sub analysis of patients with age $>55 y(n=230)$

\begin{tabular}{|c|c|c|c|c|c|c|}
\hline & \multicolumn{3}{|c|}{ Sub analysis of patients with age $>55 y(\mathrm{n}=230)$} & & & \\
\hline & $(n=77)$ & $(n=75)$ & $(n=78)$ & & & \\
\hline \multicolumn{7}{|l|}{ Systolic BP } \\
\hline Baseline & $144.0 \pm 14.4$ & $147.0 \pm 14.9$ & $145.8 \pm 16.7$ & & & \\
\hline Week 24 & $136.1 \pm 14.1$ & $131.8 \pm 15.7$ & $131.5 \pm 16.5$ & & & \\
\hline Change $^{\mathrm{c}}$ & $-7.6 \pm 17.5$ & $-14.4 \pm 16.2$ & $-15.0 \pm 22.3$ & 0.04 & 0.03 & 0.69 \\
\hline$P$-values for differences within group ${ }^{d}$ & $<0.01$ & $<0.01$ & $<0.01$ & & & \\
\hline
\end{tabular}

Abbreviations: BP, blood pressure; ITT, intention-to-treat.

Data are shown as mean \pm s.d.

a Last observation carried-forward rule applied for missing values.

${ }^{\mathrm{b}} P$-value from an ANCOVA (or ranked ANCOVA) for continuous outcomes comparing a difference between the two intervention groups (using covariates as site and baseline).

'Indicates the estimated mean change in 24-week outcome from baseline outcome.

${ }^{\mathrm{d}} P$-value from a paired $t$-test(or Wilcoxon signed rank test) within the group.

decrease in the average home SBP (a change from the 1st week of enrollment to the last week of study) compared with that in group 1 (group 1: $-0.3 \pm 12.4 \mathrm{~mm} \mathrm{Hg}$, group 2: $-0.9 \pm 14.6 \mathrm{~mm} \mathrm{Hg}$, group 3: $-5.9 \pm 15.1 \mathrm{~mm} \mathrm{Hg}, P=0.02$ for group 1 vs. $3 ; P=0.07$ for group 2 vs. 3 , Supplementary Table 2). In addition, significantly more patients in the groups 2 and 3 had the number of their BP medications decreased over the course of the investigation compared with that of the patients in group 1 (Supplementary Table 3). Last, there were no differences in the number of adverse events among the three study groups (Supplementary Table 4).

\section{DISCUSSION}

The recently published 2013 ESH/ESC guidelines for the management of arterial hypertension state that various communication technologies might theoretically be advantageous in improving BP control by having a favorable effect on improving self-monitoring, adherence and feedback from healthcare personnel. ${ }^{6}$ The effectiveness of remote monitoring has not been shown in a randomized clinical trial. ${ }^{4,12-14}$ In this context, several key findings from this study should be mentioned.

The first key finding from this study is that this study is the first to demonstrate that remote monitoring alone (group 2) or remote monitoring coupled with remote physician care (group 3) was as efficacious as the usual office care for reducing BP in patients with hypertension. To our knowledge, this study is the first to demonstrate the safety and efficacy of remote physician care in reducing BP. Although the Electronic Communications and Home Blood Pressure Monitoring Study demonstrated that web communications with remote pharmacist care is associated with significantly better target $\mathrm{BP}$ achievement at 1 year, this is the first to randomize the effectiveness of remote physician care in reducing BP. ${ }^{15,16}$ In large countries, this finding is important for the care of hypertensive patients who reside in sparsely populated regions and have limited access to healthcare. The initial hypothesis was that remote monitoring with or without remote physician care would reduce BP more significantly than usual office care. The reason for the lack of difference in the primary end point might be because the usual care group patients in this study were instructed to measure their BP at home. The EPACUM-HTA study demonstrated that home BP monitoring is associated with a $92 \%$ drug compliance rate compared with $74 \%$ in the control group. ${ }^{2}$ The high drug-adherence rate in the control group in this study (Table 3 ) supports this data and might influence the negative result for the primary end point. Second, this work was a relatively large, randomized study to assess the effectiveness of remote patient monitoring/remote physician care compared with that of usual care in reducing $\mathrm{BP}$, which includes home BP monitoring. Although we observed no significant difference in the 6month FU sitting SBP (the primary end point), there was a significant benefit in the target BP achievement and the sitting SBP reduction in the subjects over the age of 55. Although efficacy was demonstrated in a subgroup analysis, the results have merit in that they demonstrate the clinical efficacy of remote patient monitoring in a randomized clinical trial. The significant efficacy in the subgroup of patients over the age of 55 might be explained by the significantly better adherence to the home healthcare device, as demonstrated by the significantly higher number of home BP measurements in the group of patients over 55 years age (Supplementary Table 5). The lack of a significant difference in the average daytime SBP of ABPM is difficult to explain. This phenomenon is not explained by the white-coat effect because there was an equal distribution of this variable among the three study groups. The significant difference in the average home BP, which has previously been demonstrated to be well correlated with ABPMmeasured BP, supports the efficacy of remote monitoring in reducing BP. That the FU ABPM data was available in only $72.7 \%$ of the study population might have confounded the results. Our data show that a significant proportion of the subjects in the remote monitoring and remote treatment group reduced the number of $\mathrm{BP}$ medications they 


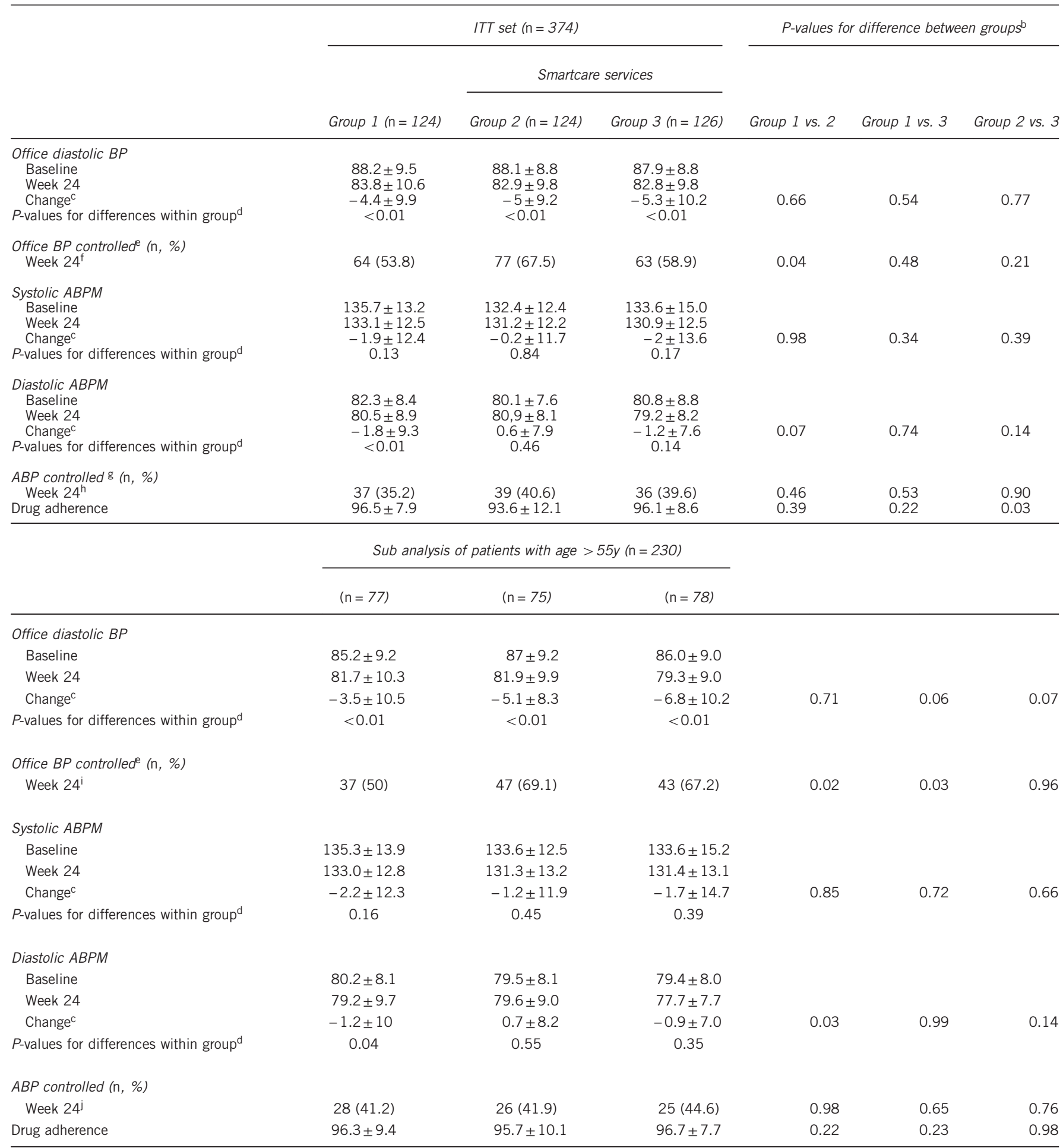

Abbreviations: ABPM, Ambulatory blood pressure monitoring; BP, blood pressure; ITT, intention-to-treat.

Data are shown as mean \pm s.d.

a Last observation carried-forward rule applied for missing values.

b P-value from an ANCOVA (or ranked ANCOVA) for continuous outcomes and Cochran-Mantel-Haenszel test for binary outcomes comparing a difference between the two intervention groups (using covariates as site and baseline).

CIndicates the estimated mean change in 24-week outcome from baseline outcome.

d $P$-value from an paired $t$-test(or Wilcoxon signed rank test) within the group.

eDefined as systolic and diastolic BP $<140 / 90 \mathrm{~mm} \mathrm{Hg}(130 / 80$ for DM and/or CKD), respectively.

${ }^{\mathrm{f}}$ Missing data for the patients 5,10 and 19 in group 1 , group 2 and group 3 , respectively.

gDefined as systolic and diastolic ABPM $<135 / 85 \mathrm{~mm} \mathrm{Hg}$ (130/80 for DM and/or CKD), respectively.

hMissing data for the patients 19,28 and 35 in group 1 , group 2 and group 3 , respectively.

'Missing data for the patients 3, 7 and 14 in group 1, group 2 and group 3, respectively.

jMissing data for the patients 9,13 and 22 in group 1 , group 2 and group 3, respectively. 
Table 4 Subgroup analysis of ABPM data in patients with true uncontrolled hypertension ${ }^{\mathrm{a}}$

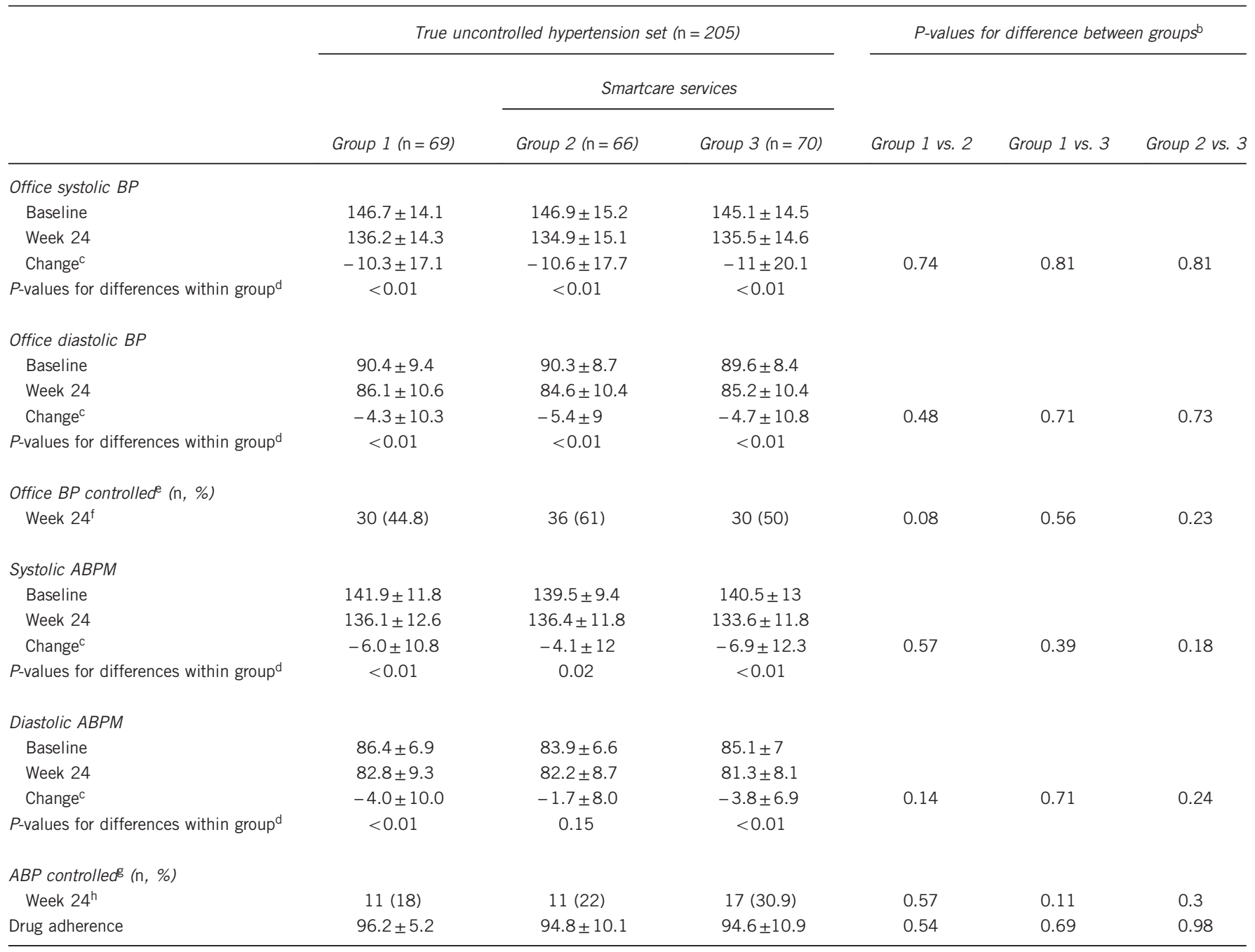

Abbreviations: ABPM, Ambulatory blood pressure monitoring; BP, blood pressure.

Data are shown as mean \pm s.d.

Data are shown as mean \pm S.d.
aLast observation carried-forward rule applied for missing values.

b $P$-value from an ANCOVA (or ranked ANCOVA) for continuous outcomes and Cochran-Mantel-Haenszel test for binary outcomes comparing a difference between the two intervention groups (using covariates as site and baseline).

cIndicates the estimated mean change in 24-week outcome from baseline outcome.

d $P$-value from a paired $t$-test (or Wilcoxon signed rank test) within the group.

e Defined as systolic and diastolic BP $<140 / 90 \mathrm{~mm} \mathrm{Hg}$ (130/80 for DM and/or CKD), respectively.

${ }^{f}$ Missing data for the patients 2, 7 and 10 in group 1, group 2 and group 3, respectively.

gDefined as systolic and diastolic ABPM $<135 / 85 \mathrm{~mm} \mathrm{Hg}$ (130/80 for DM and/or CKD), respectively.

${ }^{\mathrm{h}}$ Missing data for the patients 8,16 and 15 in group 1 , group 2 and group 3 , respectively.

were taking by the FU. This finding is in agreement with that of a previous study, which showed that home BP monitoring reduces the number of medications taken by the patients compared to the number of medications taken by the patients in the control group. ${ }^{17}$ This study is the first to show that BP medication use could be reduced by remote monitoring.

The third important finding results from our use of home BP monitoring in the control arm. Home BP monitoring is gaining wide acceptance for the treatment of arterial hypertension, with studies showing that up to $70 \%$ of hypertensive patients regularly check their BP using an office BP measuring device. ${ }^{3,18,19-21}$ Home BP monitoring has been demonstrated to improve HTN control, as well as have a strong predictive value for future cardiovascular events. ${ }^{20-22}$ We used the home BP monitoring arm as the control group to more accurately reflect real-world clinical practice, as well as to determine whether remote monitoring with or without remote physician monitoring has additional benefits in treating hypertension.

There are several limitations to this study. First, the study was initially designed to enroll 495 patients and equally randomize this sample into the three intervention groups. With a sample size of 165 patients per group, the trial was designed to have an $80 \%$ power to detect ( $\alpha=0.05$; two-sided test), with an assumption of a $20 \%$ dropout rate. The study as designed needed at least 396 study subjects. The final actual enrollment of 374 subjects results in this study being marginally underpowered. The second limitation is that we have no plausible explanation for the efficacy of remote monitoring/remote physician care in reducing office SBP and average home BP in subjects over the age of 55. Although, in theory, remote monitoring should have improved the drug adherence of the study population, there was no significant difference between the three study groups in terms of 
anti-hypertensive drug adherence (Table 3). We postulate that remote monitoring of and feedback regarding the data on the patient BP, diet and exercise patterns might have a beneficial effect in reducing BP. In conclusion, this clinical trial assessed the effectiveness of web-based remote patient monitoring with or without remote physician care (LG Smart Care) in reducing office BP in patients with hypertension. We found no significant differences in terms of the change in the mean SBP in the 24-week FU (primary outcome). The subgroup analysis revealed that patients over the age of 55 , who received remote patient monitoring, with or without remote physician care, had a significant decrease in their SBP at the 24-week FU. The web-based remote monitoring and remote physician care of hypertension was safe, with no significant increases in adverse events compared with those in the control group.

\section{ACKNOWLEDGEMENTS}

This study was supported by a 2010 consignment research grant from LG Electronics through the Ministry of Trade, Industry and Energy of South Korea (1003518). This paper was previously presented at the 2013 Korean Circulation Society Scientific meeting in Koyang City, South Korea.

1 Egan BM, Zhao Y, Axon RN. US trends in prevalence, awareness, treatment, and control of hypertension, 1988-2008. JAMA 2010; 303: 2043-2050.

2 Marquez-Contreras E, Martell-Claros N, Gil-Guillen V, de la Figuera-Von Wichmann M, Casado-Martinez JJ, Martin-de Pablos JL, Figueras M, Galera J, Serra A. Efficacy of a home blood pressure monitoring programme on therapeutic compliance in hypertension: the EAPACUM-HTA study. J Hypertens 2006; 24: 169-175.

3 Parati G, Omboni S. Role of home blood pressure telemonitoring in hypertension management: an update. Blood Press Monit 2010; 15: 285-295.

4 Parati G, Omboni S, Albini F, Piantoni L, Giuliano A, Revera M, Illyes M, Mancia G. Home blood pressure telemonitoring improves hypertension control in general practice. The TeleBPCare study. J Hypertens 2009; 27: 198-203.

5 Verdecchia P, Angeli F, Poeta F, Reboldi GP, Borgioni C, Pittavini L, Porcellati C. Validation of the A\&D UA-774 (UA-767Plus) device for self-measurement of blood pressure. Blood Press Monit 2004; 9: 225-229.

6 Mancia G, Fagard R, Narkiewicz K, Redon J, Zanchetti A, Bohm M, Christiaens T, Cifkova R, De Backer G, Dominiczak A, Galderisi M, Grobbee DE, Jaarsma T, Kirchhof $P$, Kjeldsen SE, Laurent $S$, Manolis AJ, Nilsson PM, Ruilope LM, Schmieder RE, Sirnes PA, Sleight P, Viigimaa M, Waeber B, Zannad F. 2013 ESH/ESC Guidelines for the management of arterial hypertension: the task force for the management of arterial hypertension of the European Society of Hypertension (ESH) and of the European Society of Cardiology (ESC). J Hypertens 2013; 31: 1281-1357.

7 Mancia G, De Backer G, Dominiczak A, Cifkova R, Fagard R, Germano G, Grassi G, Heagerty AM, Kjeldsen SE, Laurent S, Narkiewicz K, Ruilope L, Rynkiewicz A, Schmieder RE, Boudier HA, Zanchetti A, Vahanian A, Camm J, De Caterina R, Dean V, Dickstein K, Filippatos G, Funck-Brentano C, Hellemans I, Kristensen SD, McGregor K, Sechtem U, Silber S, Tendera M, Widimsky P, Zamorano JL, Erdine S, Kiowski W, Agabiti-Rosei E, Ambrosioni E, Lindholm LH, Viigimaa M, Adamopoulos S, Bertomeu V, Clement D, Farsang C, Gaita D, Lip G, Mallion JM, Manolis AJ, Nilsson PM, O'Brien E, Ponikowski P, Redon J, Ruschitzka F, Tamargo J, van Zwieten P, Waeber B, Williams B. 2007 guidelines for the management of arterial hypertension: the task force for the management of arterial hypertension of the European Society of Hypertension (ESH) and of the European Society of Cardiology (ESC). J Hypertens 2007; 25: 1105-1187.

8 Chobanian AV, Bakris GL, Black HR, Cushman WC, Green LA, Izzo JL Jr, Jones DW, Materson BJ, Oparil S, Wright JT Jr., Roccella EJ. Seventh report of the joint national committee on prevention, detection, evaluation, and treatment of high blood pressure. Hypertension 2003; 42: 1206-1252.

9 Svetkey LP, Pollak KI, Yancy WS Jr, Dolor RJ, Batch BC, Samsa G, Matchar DB, Lin $\mathrm{PH}$. Hypertension improvement project: randomized trial of quality improvement for physicians and lifestyle modification for patients. Hypertension 2009; 54: 1226-1233.

10 Park MJ, Kim HS, Kim KS. Cellular phone and Internet-based individual intervention on blood pressure and obesity in obese patients with hypertension. Int J Med Inform 2009; 78: 704-710.

11 Bosworth HB, Olsen MK, Grubber JM, Neary AM, Orr MM, Powers BJ, Adams MB, Svetkey LP, Reed SD, Li Y, Dolor RJ, Oddone EZ. Two self-management interventions to improve hypertension control: a randomized trial. Ann Intern Med 2009; 151 . 687-695.

12 Shea K, Chamoff B. Telehomecare communication and self-care in chronic conditions: moving toward a shared understanding. Worldviews Evid Based Nurs 2012; 9: 109-116.

13 Neumann CL, Menne J, Rieken EM, Fischer N, Weber MH, Haller H, Schulz EG. Blood pressure telemonitoring is useful to achieve blood pressure control in inadequately treated patients with arterial hypertension. J Hum Hypertens 2011; 25: 732-738.

14 Omboni S, Guarda A. Impact of home blood pressure telemonitoring and blood pressure control: a meta-analysis of randomized controlled studies. Am J Hypertens 2011; 24: 989-998.

15 Staessen JA, Den Hond E, Celis H, Fagard R, Keary L, Vandenhoven G, O'Brien ET. Antihypertensive treatment based on blood pressure measurement at home or in the physician's office: a randomized controlled trial. JAMA 2004; 291 . 955-964.

16 Green BB, Cook AJ, Ralston JD, Fishman PA, Catz SL, Carlson J, Carrell D, Tyll L, Larson EB, Thompson RS. Effectiveness of home blood pressure monitoring, web communication, and pharmacist care on hypertension control: a randomized controlled trial. JAMA 2008; 299: 2857-2867.

17 Verberk WJ, Kroon AA, Lenders JW, Kessels AG, van Montfrans GA, Smit AJ, van der Kuy PH, Nelemans PJ, Rennenberg RJ, Grobbee DE, Beltman FW, Joore MA, Brunenberg DE, Dirksen C, Thien T, de Leeuw PW. Self-measurement of blood pressure at home reduces the need for antihypertensive drugs: a randomized, controlled trial. Hypertension 2007; 50: 1019-1025.

18 Logan AG, Dunai A, Mclsaac WJ, Irvine MJ, Tisler A. Attitudes of primary care physicians and their patients about home blood pressure monitoring in Ontario. J Hypertens 2008; 26: 446-452.

19 Cuspidi C, Meani S, Fusi V, Salerno M, Valerio C, Severgnini B, Catini E, Leonetti G, Magrini $F$, Zanchetti A. Home blood pressure measurement and its relationship with blood pressure control in a large selected hypertensive population. J Hum Hypertens 2004; 18: 725-731.

20 Agarwal R, Bills JE, Hecht TJ, Light RP. Role of home blood pressure monitoring in overcoming therapeutic inertia and improving hypertension control: a systematic review and meta-analysis. Hypertension 2011; 57: 29-38.

21 Imai Y, Obara T, Asamaya K, Ohkubo T. The reason why home blood pressure measurements are preferred over clinic or ambulatory blood pressure in Japan. Hypertens Res 2013; 36: 661-672.

22 Aparicio LS, Thijs L, Asayama K, Barochiner J, Boggia J, Gu YM, Cuffaro PE, Liu YP, Niiranen TJ, Ohkubo T, Johansson JK, Kikuya M, Hozawa A, Tsuji I, Imai Y, Sandoya E, Stergiou GS, Waisman GD, Staessen JA. International Database on home blood pressure in relation to cardiovascular outcome (IDHOCO) investigators. Reference frame for home pulse pressure based on cardiovascular risk in 6470 subjects from 5 populations. Hypertens Res 2014; 37: 672-678.

Supplementary Information accompanies the paper on Hypertension Research website (http://www.nature.com/hr) 